Draft VERSion April 27, 2021

Typeset using LATEX twocolumn style in AASTeX63

\title{
Multimessenger signals from black hole-neutron star mergers without significant tidal disruption
}

\author{
William E. East,${ }^{1}$ Luis Lehner,${ }^{1}$ Steven L. Liebling, ${ }^{2}$ and Carlos Palenzuela ${ }^{3}$ \\ ${ }^{1}$ Perimeter Institute, 31 Caroline Street, Waterloo, ON N2L 2Y5, Canada \\ ${ }^{2}$ Long Island University, Brookville, New York 11548, USA \\ ${ }^{3}$ Departament de Física \& IAC3, Universitat de les Illes Balears, Palma de Mallorca, Baleares E-07122, Spain
}

\begin{abstract}
We study the multimessenger signals from the merger of a black hole with a magnetized neutron star using resistive magnetohydrodynamics simulations coupled to full general relativity. We focus on a case with a 5:1 mass ratio, where only a small amount of the neutron star matter remains post-merger, but we nevertheless find that significant electromagnetic radiation can be powered by the interaction of the neutron star's magnetosphere with the black hole. In the lead-up to merger, strong twisting of magnetic field lines from the inspiral leads to plasmoid emission and results in a luminosity in excess of that expected from unipolar induction. We find that the strongest emission occurs shortly after merger during a transitory period in which magnetic loops form and escape the central region. The remaining magnetic field collimates around the spin axis of the remnant black hole before dissipating, an indication that, in more favorable scenarios (higher black hole spin/lower mass ratio) with larger accretion disks, a jet would form.
\end{abstract}

\section{INTRODUCTION}

The observations by gravitational wave (GW) detectors and conventional electromagnetic telescopes of the binary neutron star (NS) merger known as GW170817 provided a spectacular inauguration of the nascent enterprise of multimessenger astronomy (Abbott et al. 2017a,b). by constraining the equation of state of high density matter, establishing that NS mergers source short gamma ray bursts, and demonstrating the production of heavy elements from such mergers through observations of the subsequent kilonova. At the same time, nonvacuum binaries present challenges to current GW observatories. In the case of binary NS systems, the lower total mass means that the stars come into contact at GW frequencies above a kilohertz, where current detectors rapidly lose sensitivity.

Black hole-neutron star (BHNS) binaries can potentially have much larger masses than binary NSs, and thus their merger is, in principle, easier to detect, as it takes place at lower frequencies where current detectors are more sensitive $\left[f \gtrsim 600\left(10 M_{\odot} / M_{T}\right) \mathrm{Hz}\right]$. Importantly, for high enough binary mass ratios, the NS might be devoured by the black hole $(\mathrm{BH})$ without being disrupted, making the GW signal essentially indistinguishable from those sourced by a binary $\mathrm{BH}$ with the same masses.

For a sufficiently low mass ratio (depending on the $\mathrm{BH}$ spin and NS equation of state), the star is tidally disrupted outside the effective innermost stable orbit of the binary, resulting in the ejection of material from the system and the formation of an accretion disk that may produce an electromagnetic counterpart, in particular a short gamma ray burst. This scenario would be ideal for producing signals that would probe the strong gravitational field (and arguably the strongest curvatures) as well as the properties of high density matter.

However, observations of stellar mass BHs in binaries obtained to date (both in electromagnetic observations of LMXBs and GW events by $\mathrm{LIGO/Virgo)}$ suggest a dearth of such low mass ratio systems (Abbott et al. 2020a; Corral-Santana et al. 2016). Instead, the evidence suggests a prevalence of higher mass BHs ( $\left.66 M_{\odot}\right)$ with low spins. While this may be a result of observational bias, and the companions in these binaries were not NSs (with one or two possible exceptions), this suggests that disruption or other tidal effects might not be strong enough to manifest in the GWs from most BHNS with high signal-to-noise, or to lead to an accretion powered transient. For instance, the recent event GW190814 (Abbott et al. 2020b), with mass ratio of $\approx 10: 1$ had a companion mass in the range of $\approx 2.50-2.67 M_{\odot}$. The GW signal does not indicate whether the companion was an NS or a $\mathrm{BH}$, and its mass does not favor either one in particular unless further (potentially biased) assumptions are made. Thus, this object was either the most massive NS, or the lightest $\mathrm{BH}$, yet observed. Either answer would have profound consequences for astrophysics and nuclear physics. 
The unknown nature of the secondary object in GW190814 demonstrates the importance of understanding potential electromagnetic signals that could break such degeneracies. Because the NS in a BHNS is likely magnetized (Spruit 2008), its interaction with a BHwhich intensifies as the orbit tightens - could give rise to electromagnetic counterparts. Several authors have discussed how this might occur within a unipolar induction (UI) model (Hansen \& Lyutikov 2001; McWilliams \& Levin 2011; Piro 2012; Lai 2012; D'Orazio et al. 2016), with the BH acting as a battery in a DC circuit with the NS. However, whether this simple steady-state picture is accurate remains an open question. In particular, one interesting possibility that has not been explored in detail is that the continuous twisting of magnetic field lines leads to more complicated reconnection and plasmoid ejection, as well as emission channeled through the development of a current sheet. The twisting angle is related to the $\mathrm{BH}$ velocity relative to the NS as $\zeta_{\phi} \approx 4 v_{\text {rel }} /(\pi c$ ) (Lai 2012), and thus one expects these effects, and the departure from the UI model, to be strongest around merger.

Several studies of the dynamics of magnetospheric interactions in binary NS mergers have demonstrated how the binary's kinetic energy can be converted into electromagnetic radiation (Palenzuela et al. 2013b,a; Ponce et al. 2014) through UI and accelerating magnetic dipole effects (Carrasco \& Shibata 2020), as well as how the twisting of magnetic flux tubes can produce periodic flaring (Most \& Philippov 2020).

The BHNS case has been studied in the force-free approximation assuming a helical Killing vector (Paschalidis et al. 2013; Carrasco et al. 2021), which would approximately hold during the early inspiral. Here, we concentrate on the final, most dynamical stage of a 5:1 mass-ratio BHNS merger using full GR simulations. This allows us to explore the interaction of the $\mathrm{BH}$ with the NS's magnetosphere when it is strongest, as well as the post-merger dissipation of the magnetosphere, which may also source an electromagnetic transient (Lehner et al. 2012; Lyutikov \& McKinney 2011; Pan \& Yang 2019). We treat the plasma with a resistive magnetohydrodynamics (MHD) approach (Palenzuela 2013) that can interpolate between the fluid pressure dominated regions inside the NS, and the magnetically dominated regime in the tenuous plasma surrounding the binary.

We find that the interaction of the BH with the NS's magnetic field during merger leads to significant electromagnetic emission. The continual twisting of the magnetic field produces current sheets with a complex configuration, occurring both in the vicinity of the $\mathrm{BH}$ and also at larger distances. These current sheets, where charges can be effectively accelerated, result as field lines are stretched, forming X-points where reconnection takes place. Plasmoids, isolated regions of closed field lines, are produced from the reconnection, resulting in a level of electromagnetic emission stronger than that estimated by UI.

\section{SETUP}

To study the system of interest, we implement the general relativistic, resistive MHD equations, coupled to Einstein gravity. Thus, we capture the behavior of both resistive, magnetized matter, and its interplay with the dynamical spacetime. More details on the numerical methods and implementation are given in the appendix. Unless otherwise stated, in the following we use LorentzHeaviside units with $G=c=1$.

We adopt initial data describing a nonspinning, quasicircular binary consisting of a $\mathrm{BH}$ with mass $M_{\mathrm{BH}}=$ $7 M_{\odot}$ and an NS with mass $M_{\mathrm{NS}}=1.33 M_{\odot}$, constructed with the Lorene library (Gourgoulhon et al. 2010). The NS obeys a polytropic equation of state $p=K \rho^{\Gamma}$ with adiabatic index $\Gamma=2$ and has a radius of $R_{\mathrm{NS}}=11.62 \mathrm{~km}$. The initial orbital frequency is $\Omega=890 \mathrm{~Hz}$, and the binary undergoes roughly 2.5 orbits before merging. Choosing a mass ratio that allows a small amount of matter to remain bound but outside the $\mathrm{BH}$ for some time enables us to study the development of magnetic field structures post-merger that would be more marked, and relevant for observations, in cases with lower mass ratios/higher $\mathrm{BH}$ spins.

The NS is given a dipole magnetic field with surface strength $B_{*}=3 \times 10^{9} \mathrm{G}$, though at this low value the magnetic field does not have any significant effect on the spacetime (Ioka \& Taniguchi 2000), nor on the hydrodynamics, except perhaps for the low density material in the vicinity of the post-merger $\mathrm{BH}$. Hence our results can be approximately scaled to arbitrary NS magnetic field values $B_{9}:=B_{*} /\left(10^{9} \mathrm{G}\right)$ within this regime.

\section{RESULTS}

The BHNS binary undergoes roughly two orbits before the outermost layer of the star is stripped away and the bulk of the star is swallowed by the $\mathrm{BH}$. This results in a final $\mathrm{BH}$ with dimensionless $\operatorname{spin} a / M \simeq 0.4$. Only a small fraction of the matter remains outside the $\mathrm{BH}$ after merger $\left(\approx 5 \% M_{\mathrm{NS}}\right)$, and it is slowly accreting onto the $\mathrm{BH}$ at a rate $\dot{M} \propto t^{-5 / 3}$ (there is negligible ejecta). While most of this behavior had been understood previously (e.g. Chawla et al. 2010), our main focus here is on examining the behavior of the electromagnetic field and the potential electromagnetic signals induced by the merger. 
Of particular relevance is the structure of current sheets associated with the system, which not only bears a strong correlation with the dynamics of the binary (as already indicated in Palenzuela et al. 2013b,a; Ponce et al. 2014; Most \& Philippov 2020), but also with the characteristics of the compact objects involved. Figure 1 illustrates this structure, showing current sheets that have developed both on and off the orbital plane at two representative times prior to merger. Roughly, we can understand the development of these current sheets as due to two different effects. On the one hand, field lines emanating from the NS get sufficiently bent, as the star orbits, to seed a current sheet some distance away, even though the NS is not spinning as in previous binary NS studies. Analogous to the light-cylinder radius of an isolated spinning NS, we expect this to occur at a lengthscale $L \simeq \Omega^{-1}$ (where $\Omega$ is the angular velocity of the binary). On the other hand, field lines sufficiently close to the $\mathrm{BH}$ get twisted to such a large degree as to seed a current sheet in the wake of the BH's trajectory, even when the $\mathrm{BH}$ is not spinning (also previously observed in simulations; Palenzuela et al. 2010; Neilsen et al. 2011). As evident in Fig. 1, this latter current sheet is smaller scale, comparable to the radius of the $\mathrm{BH}$.

Because current sheets are the site of reconnection, their dynamics is key to understanding the electromagnetic output of the system. As the orbit proceeds, field lines are more rapidly wound, and the magnetic field strength in the strongly gravitating vicinity of the $\mathrm{BH}$ increases. In this low density region, our resistive scheme approaches the force-free limit. With sufficient winding, X-point reconnection occurs and leads to closed field loops that propagate away at near-luminal speeds, with loops forming near the $\mathrm{BH}$ having greater field strength than those produced further away ${ }^{1}$. Previous studies of reconnection in the force-free approximation have found that the process is fast (relative, say, to near the ideal MHD limit), with relativistic speeds roughly $v_{\text {rec }} \approx 0.1$ (Lehner et al. 2012; Parfrey et al. 2013). In particular, the reconnection speed is not set by the timescale of bulk dissipation since in regions that are nearly force-free there is little Joule heating at the current sheets.

Examining the total Poynting flux from the system, shown at two different spheres of observation in Fig. 2, we can see strong modulations at several different frequencies in the lead-up to merger, which we can at-

\footnotetext{
${ }^{1}$ Greater field strength of loops formed near the BH has also been observed in force-free solutions sourced by an effective NS orbiting in a fixed Kerr BH spacetime (Carrasco et al. 2021).
}
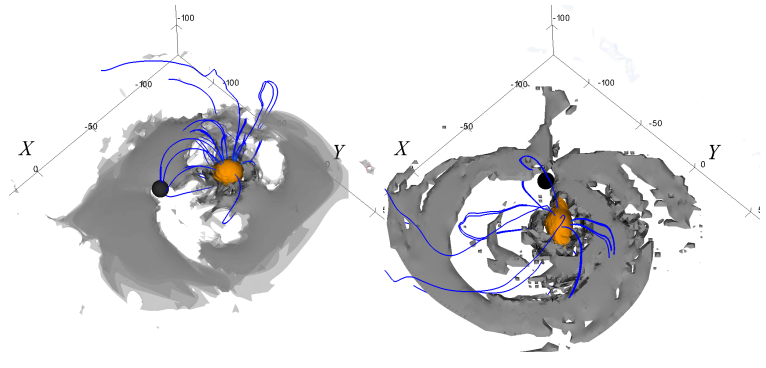

Figure 1. Snapshots at times $t=6.4$ (left) and $8.4 \mathrm{~ms}$ (right). The figure illustrates the star (yellow, isopycnic contour at $\rho=3 \times 10^{11} \mathrm{~g} / \mathrm{cm}^{3}$ ), the $\mathrm{BH}$ (black), magnetic field lines (blue, integrated over some seeds centered on the star), and current sheet structure (defined by the norm of the current $J_{i} J^{i}$; gray, semitransparent). The system orbits counterclockwise. The snapshots demonstrate the significant dynamics of the electromagnetic field, particularly the structure of the current sheets. A current sheet forms behind the high curvature region of the $\mathrm{BH}$, and trails the $\mathrm{BH}$ as it orbits. Another current sheet is correlated with the motion of the NS, but at some distance from the star. As the orbit proceeds, significant deformation of field lines leads to more current sheets. These sheets support closed magnetic field loops that arise from magnetic reconnection and that are transported away from the system.

tempt to associate with the abovementioned features of the current sheets. We expect high frequency features associated with the $\mathrm{BH}$ current sheet on timescales of $\sim R_{\mathrm{BH}} / v_{\text {rec }} \approx 0.5 \mathrm{~ms}$, while we expect lower frequency modulations due to the more distant current sheets associated with NS orbit on timescales of $\sim \Omega^{-1} / v_{\text {rec }} \approx 6$ ms (using $v_{\text {rec }} \approx 0.1$ and $\Omega \approx 1.6 \mathrm{kHz}$ ). This is consistent with the variation in the electromagnetic flux seen in Fig. 2, though the latter is admittedly rather noisy due to the complicated plasma dynamics.

The system radiates both in electromagnetic and gravitational channels, though the GW signal is dependent only on the acceleration of the source's quadrupole (and not the magnetic field strength or reprocessing of electromagnetic outflows). The inset of Fig. 2 illustrates the plus polarization of the GWs. The familiar chirp is present, and the post-merger, quasinormal ringing is consistent with the expectation for a remnant $\mathrm{BH}$ with $M \simeq 8.3 M_{\odot}$ and $a / M \simeq 0.4$ (as predicted from simple arguments Foucart 2012; Buonanno et al. 2008).

Interestingly, the peak of the electromagnetic emission occurs a couple of milliseconds later than that of the GWs. While the GW peak corresponds roughly to the maximum rate of change of the system's quadrupole moment, the electromagnetic emission arises from the reconnection of magnetic field lines. Thus the time delay of the electromagnetic emission is a consequence of the magnetic reconfiguration forced by the formation of 
a common horizon for the $\mathrm{BH}$ and NS. We also note that the delay is commensurate with the spin period of the remnant $\mathrm{BH}$, roughly $2.5 \mathrm{~ms}$.

An important result of this paper is the comparison of this luminosity with that predicted by the UI model $L_{\mathrm{UI}}$, which we also include in Fig. 2. Recall that the UI model prediction for the binary studied here is $L_{\mathrm{UI}} \approx 4 \times 10^{34}\left(v_{\text {rel }} / c\right)^{2} B_{9}^{2}(100 \mathrm{~km} / d)^{6} \mathrm{erg} / \mathrm{s}$. We use the Keplerian expressions for $\{v, r\}$ in terms of the orbital frequency $\Omega$. We estimate this latter one in terms of the GW frequency $f \approx \Omega \pi$ (hence $L_{\mathrm{UI}} \propto f^{14 / 3}$ ), thus capturing the more rapid rate at which $\{v, r\}$ change due to the increasingly strong gravitational effects as the final plunge approaches. The simulations consistently show a larger luminosity than that of the UI model at earlier times (lower frequencies), as that model does not capture the complex phenomena associated with reconnections and the role of current sheets. Close to the coalescence, however, the two become nearly equal ${ }^{2}$. Furthermore, notice that a slightly weaker Poynting flux is measured at larger extraction spheres, reflective of the energy dissipated at current sheets located between the spheres (a behavior recently pointed out in Most \& Philippov 2020). Pre-merger, this integrated difference corresponds to a dissipated energy of roughly $2 \times 10^{32} B_{9}^{2}$ erg (this includes accounting for the energy stored in the region between the extraction spheres, which is subdominant). Post-merger, this difference is more significant, though this is likely, at least in part, because the larger scale of the post-merger electromagnetic field structures means that one must go to larger radii to be in the wave zone and free of finite extraction radius effects. In the following discussion of the luminosity, we take the results from the largest radius shown.

The significant post-merger emission evident in Fig. 2 is of the order of magnitude one would calculate for the Blandford-Znajek luminosity (Blandford \& Znajek 1977) using the initial magnetic field strength of the NS. However, as discussed further below, the magnetic field in the vicinity of the final BH is significantly lower, and this emission is actually powered by the radiation of magnetic field loops. Previous studies of the collapse of a magnetized NS in force-free or ideal MHD have found that the resulting $\mathrm{BH}$ sheds its magnetosphere within a timescale $\approx 100 M_{\mathrm{BH}} \approx 4.0 \mathrm{~ms}\left(M_{\mathrm{BH}} / 8.3 M_{\odot}\right)($ Baumgarte \& Shapiro 2003; Lehner et al. 2012; Lyutikov \& McKinney 2011) or less. This is of the same order, if somewhat shorter than, the timescale over which the

\footnotetext{
${ }^{2}$ Note though that the assumptions underlying the UI estimate become increasingly suspect at merger.
}

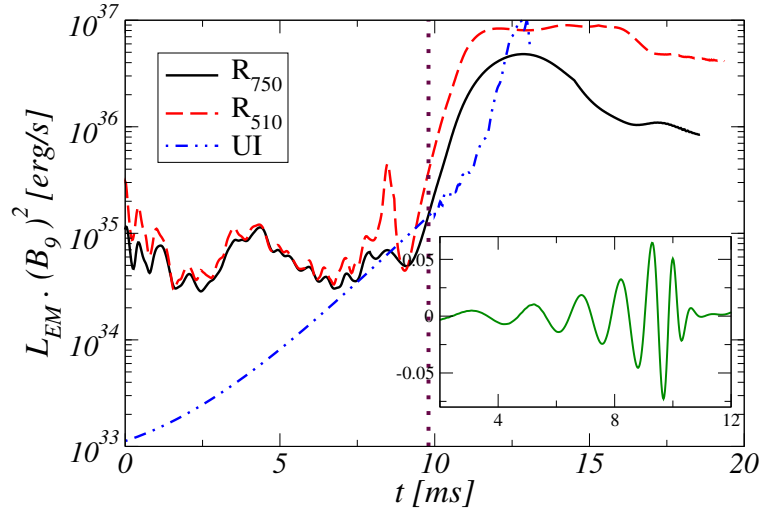

Figure 2. Poynting flux luminosity measured at extraction surfaces with radii $R_{e}=510 \mathrm{~km}$ and $750 \mathrm{~km}$ as a function of retarded time, along with the UI estimate (dashed) normalized to a surface field strength of $B=10^{9} \mathrm{G}$. The inset displays the plus polarization of the GWs (indicated by the real part of the $l=m=2$ mode of $\Psi_{4}$ ). The vertical line indicates the time at which the GW peaks. Around this time, the electromagnetic luminosity grows steeply until it also reaches a peak.

post-merger luminosity (at the largest extraction radius) decreases. One factor contributing to this longer timescale compared to NS collapse is the asymmetry of the merger scenario, which leads to magnetic field loops gathered to one side, instead of forming an axisymmetric, equatorial current sheet.

The luminosity is still significant at the end of the simulation, but cannot be long lived if it is not powered by the $\mathrm{BH}$ and/or accretion. We can obtain an upper bound on its persistance at this level using the total magnetic energy stored in the NS's initial dipole as an estimate of the available energy (ignoring that a significant amount is captured by the $\mathrm{BH}$ ) to obtain $U_{\mathrm{dip}} / L_{\mathrm{EM}} \approx 100 \mathrm{~ms}$.

To quantify the directional dependence, we compute the luminosity within specific ranges of the polar angle, normalized by their angular size. In Fig. 3 we can see that there is an increase in luminosity with polar angle, indicating greater emission near the orbital plane. The emission close to the orbital plane is also less variable than the polar emission. Near merger, the luminosity at all angles increases similarly rapidly. Post-merger, the emission from the equatorial region strongly dominates, and the luminosity at all angles then decreases as the small amount of remaining matter is accreted and magnetic field is shed or swallowed by the $\mathrm{BH}$ (see also Lehner et al. 2012).

The angular dependence is further illustrated in Fig. 4, which shows several snapshots of the angular dependence of the electromagnetic luminosity leading up to, and following merger. In addition to the stronger emis- 
sion in the vicinity of the equator, we can also see the strong nonaxisymmetric nature of the radiation. However, from the time integrated flux in the bottom panel of Fig. 4, we can see that the emission is not strongly observer dependent, in the sense that the energy emitted in most directions is within an order of magnitude of the maximum.

Finally, it is interesting to examine the large-scale magnetic field post-merger, when there is a spinning $\mathrm{BH}$ with a small amount of matter remaining outside. In Fig. 5, we display three snapshots of select magnetic field lines at times $\approx 3.5,6.5$, and $9.1 \mathrm{~ms}$ after merger (at intervals roughly corresponding to the $\mathrm{BH}$ rotation period). Most apparent, the magnetic field near the $\mathrm{BH}$ appears increasingly collimated and ordered, suggestive of possible jet formation à la the Blandford-Znajek process. However, this is only ephemeral for the system studied here because of the dearth of matter to anchor the magnetic field. What little matter remains has insufficient angular momentum and is being quickly accreted by the $\mathrm{BH}$. With nothing to arrest the accretion (e.g. Narayan et al. 2003), the field lines from the BH become increasingly vertical with diminishing strength. Instead of Blandford-Znajek emission, one can see the loops formed by equatorial currents in the vicinity of the $\mathrm{BH}$ propagate away, still producing sizeable, though more transient, emission. It would be interesting to study in more detail the near horizon plasma dynamics giving rise to this emission, though we leave this for future study.

\section{DISCUSSION}

This study focuses on a BHNS system sitting roughly at the boundary between two regimes: one in which the NS fails to disrupt with essentially no electromagnetic emission powered by post-merger matter, and the other in which the star fully disrupts leading to significant emission due to the presence of an accretion disk and/or ejecta. The latter scenario is expected only for systems with a sufficiently low mass ratio and/or high BH spina scenario so far seemingly disfavored by GW observations (assuming the binary $\mathrm{BH}$ observations made so far are representative of BHs in generic BHNSs). For such a disk, a standing question has been the degree and timescale over which the magnetic field reorders and gives rise to a configuration favoring a jet (see, e.g. Paschalidis et al. 2015; Ruiz et al. 2018; Christie et al. 2019b; Foucart 2020, ).

In our case, where only a little material remains temporarily outside the $\mathrm{BH}$, we indeed see such a reordering. Our evolutions suggest the formation post-merger of poloidal structure which is a requirement of many models of $\mathrm{BH}$ jets (e.g. Hawley et al. 2015; Christie

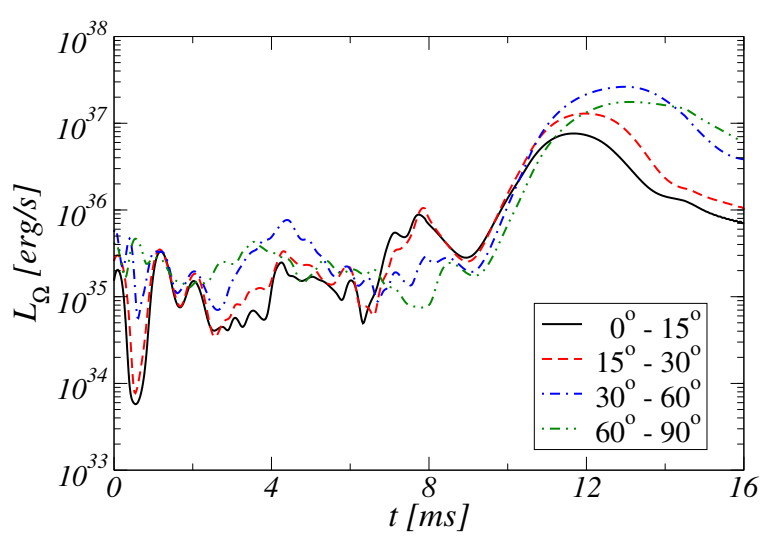

Figure 3. Luminosity at an extraction radius of $R=750$ $\mathrm{km}$ through specified ranges of the polar angle (normalized by their angular size) with $0^{\circ}$ corresponding to the direction of the total angular momentum of the system. During the inspiral phase, all regions show short timescale modulations, but the polar regions (in the direction transverse to the orbit) exhibit an overall increase that contrasts with the flatter level close to the equatorial (orbital) plane. At merger, the luminosity at all angles increases sharply, but subsequently decreases post-merger as the $\mathrm{BH}$ accretes the little remaining matter, and the magnetic field lines are shed away or swallowed by the BH.

et al. 2019a). Furthermore, we note that in principle the amount of material in such a disk need not be too large. As argued in Lee \& Ramirez-Ruiz (2007), as little as $10^{-3} M_{\odot}$ can anchor magnetic fields with strengths up to $10^{15} \mathrm{G}$. For the physical parameters studied here, such an amount of matter, however, is accreted in a relatively short timescale. The fact that the magnetic field is successfully collimated even with such a small amount of material remaining is suggestive of what would happen in more favorable configurations where the NS is disrupted earlier and the amount of post-merger matter is larger.

In the opposite direction (i.e. no spin and higher mass ratio), one would be left only with the type of electromagnetic counterparts that would be induced by the features elucidated in this work: development of a complex current sheet, strong twisting of field lines, and formation of X-points; all favoring the emission of plasmoids. As we point out, the energy associated with such structures is enhanced due to the spacetime dynamics, thus improving the observational prospects.

We comment briefly on the potential for observing signals from the system. LIGO/Virgo is able to observe GWs from such binaries at distances in excess of 100 $\mathrm{Mpc}$, and this horizon will improve with further upgrades, including dramatically with planned third generation detectors (e.g. Sathyaprakash et al. 2019). On the electromagnetic front, at high energies, and tak- 

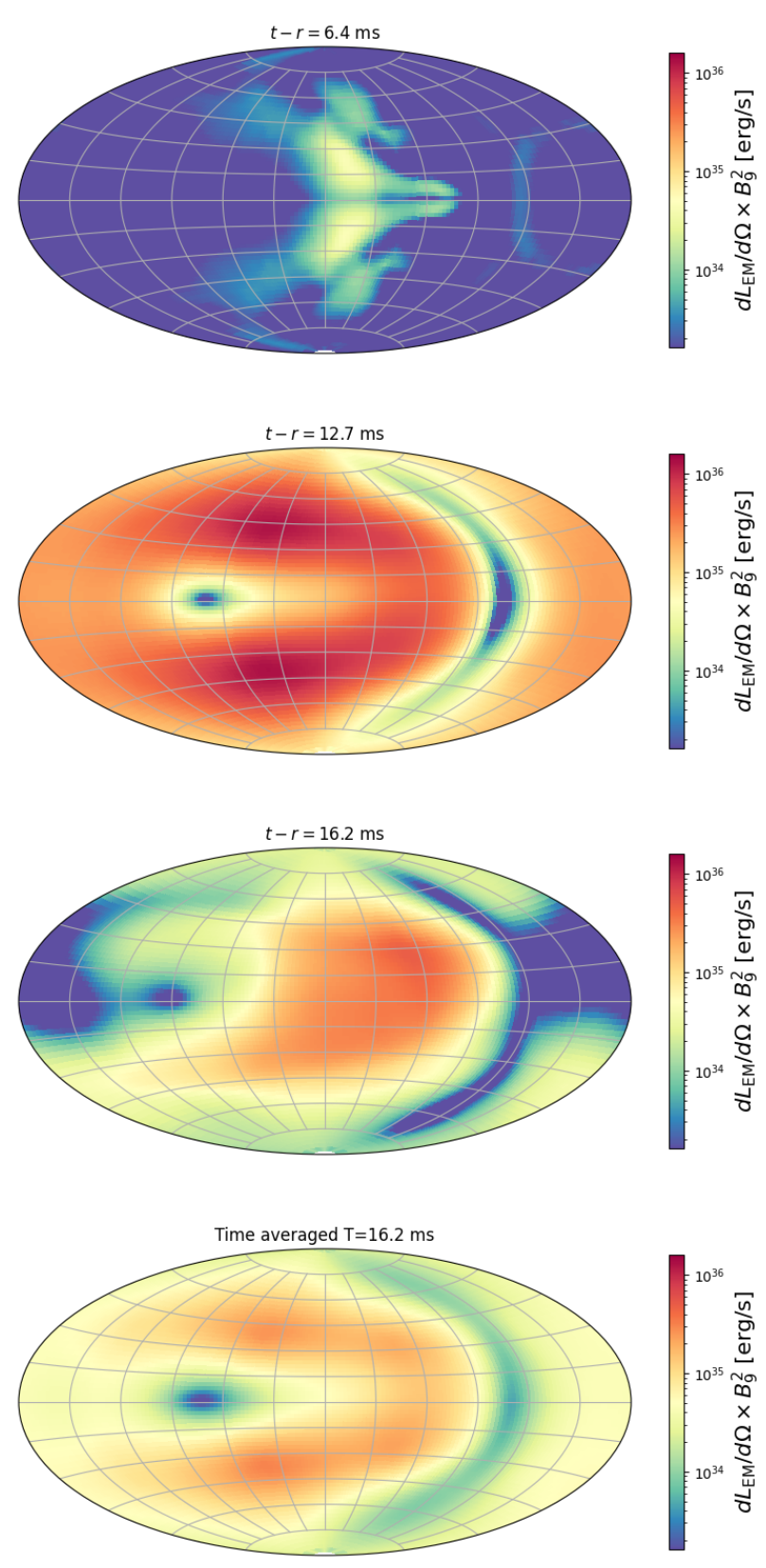

Figure 4. Snapshots of the Poynting flux at look-back times $t-r=6.4,12.7$, and $16.2 \mathrm{~ms}$ (from top to bottom) at an extraction sphere at radius $r=750 \mathrm{~km}$. The bottommost panel shows the time-averaged flux for the duration $T=16.2$ $\mathrm{ms}$, or equivalently the total radiated energy per steradian $\times 1 / T$.

ing the Burst Alert Telescope at Swift as an example, its sensitivity to $100 \mathrm{keV}$ photons (Barthelmy et al. 2005 ) would allow for a detection up to a distance of

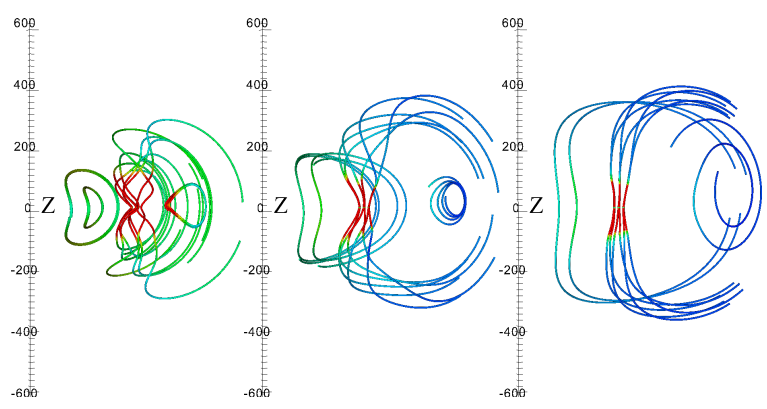

Figure 5. Snapshots of the magnetic field at times $t=13.3$, 16.4 , and $19.0 \mathrm{~ms}$ from a distant vantage point. A selection of magnetic field lines is shown, including lines passing near the $\mathrm{BH}$ (where the fields are the strongest) and a few additional lines away from the $\mathrm{BH}$, which show the emission of a plasmoid. The color indicates the magnetic field strength ranging from $10^{4} B_{9} \mathrm{G}$ to $10^{6} B_{9} \mathrm{G}$, with all panels using the same colormap. The field topology in the vicinity of the $\mathrm{BH}$ gradually collimates along the $\mathrm{BH}$ spin axis.

$D_{L} \simeq 50\left(B_{12}\right) \mathrm{Mpc}^{3}$. Such an estimate assumes perfect conversion efficiency; however, we note realistic estimates are less optimistic by a factor of $\simeq 1 \%-10 \%$ (e.g. Palmer et al. 2019).

At lower frequencies, coherent radio emission has been suggested as a more likely observational prospect. As discussed in Most \& Philippov (2020) and Sridhar et al. (2020), both magnetic reconnection in the current sheet and the synchrotron maser process due to plasmoids shocking the ambient plasma are potential mechanisms for such emission.

\footnotetext{
${ }^{3}$ Skymaps of the potential high energy radiation produced by the system will be presented elsewhere (Ortiz \& et al. 2021).
} 


\section{ACKNOWLEDGMENTS}

We thank Federico Carrasco and Alexander Philippov for discussions. LL thanks the CCA at the Flatiron Institute for hospitality during an early stage of this work. SLL is supported by the NSF under grants PHY1912769 and PHY-2011383. CP acknowledges support from the Spanish Ministry of Economy and Competitiveness grants AYA2016-80289-P and PID2019-110301GBI00 (AEI/FEDER, UE). WE and LL are supported in part by NSERC through a Discovery Grant, and LL also thanks CIFAR for support. Computations were performed on XSEDE resources and the Niagara supercomputer at SciNet. SciNet is funded by the Canada Foundation for Innovation; the Government of Ontario; Ontario Research Fund - Research Excellence; and the University of Toronto. Research at Perimeter Institute is supported by the Government of Canada and by the Province of Ontario through the Ministry of Research, Innovation and Science.

\section{APPENDIX}

In this section, we give more details on our scheme for evolving a binary with a BH and magnetized NS, including the numerical implemention. We employ the HAD computational infrastructure (HAD home page 2010) and implement the general relativistic, resistive MHD equations, as described in Palenzuela (2013) and Palenzuela et al. (2009), coupled to Einstein gravity in the CCZ4 formulation (Alic et al. 2012; Bezares et al. 2017). For a more thorough description of the implementation we refer the reader to aforementioned references.

The magnetized star is described by the total stress-energy tensor

$$
T_{\mu \nu}=[\rho(1+\epsilon)+p] u_{\mu} u_{\nu}+p g_{\mu \nu}+F_{\mu}{ }^{\lambda} F_{\nu \lambda}-\frac{1}{4} g_{\mu \nu} F^{\lambda \alpha} F_{\lambda \alpha}
$$

where $F^{\mu \nu}$ is the Faraday tensor, which can be decomposed in terms of the electric $E^{\mu}$ and magnetic $B^{\mu}$ fields ${ }^{4}$. Here $u^{a}$ is the fluid four velocity, $\rho$ is the rest mass density, $\epsilon$ the internal energy, and $p$ is the pressure. Here we use a $\Gamma=2$ equation of state $p=\rho \epsilon$.

The evolution of the magnetized matter must obey both the Maxwell equations and the conservation of total stressenergy tensor. Going beyond the ideal MHD limit, which treats the fluid as a perfect conductor, requires a prescription for the electric current to close the system of equations, called resistive MHD. The ideal MHD and the force-free limits can be captured with the phenomenological current introduced in Palenzuela (2013), which includes the isotropic conductivity and (some of) the anisotropic Hall terms

$$
J_{i}=q\left[(1-H) v_{i}+H v_{i}^{d}\right]+\frac{\sigma}{1+\zeta^{2}}\left[\mathcal{E}_{i}+\frac{\zeta^{2}}{B^{2}}\left(E^{k} B_{k}\right) B_{i}\right]
$$

where $v_{i}^{d}=\epsilon_{i j k} E^{j} B^{k} / B^{2}$ is the drift velocity, $v_{i}=u_{i} / W$ is the Eulerian velocity with associated Lorentz factor $W=\alpha u^{t}$ (in terms of the lapse $\alpha$ ), and we have introduced the shorthand

$$
\mathcal{E}_{i}=W\left[E_{i}+\epsilon_{i j k} v^{j} B^{k}-\left(v_{k} E^{k}\right) v_{i}\right] .
$$

The kernel function $H$ is defined such that it smoothly varies with density from zero inside the star, to unity outside, with a very high isotropic conductivity $\sigma=2 \times 10^{10} \mathrm{~s}^{-1}$ and an anisotropic ratio $\zeta=H \sigma \tau$, where we set $\tau=10^{5} \sigma^{-1}=$

\footnotetext{
${ }^{4}$ Notice that a factor of $1 / \sqrt{4 \pi}$ has been absorbed in the definition of the electromagnetic fields.
} 
$5 \times 10^{-6} \mathrm{~s}$ to be shorter than the dynamical time of the binary, but much longer than $\sigma^{-1}$. In our particular scenario, in the interior of the star (i.e., $H \approx 0$ ), the large isotropic conductivity effectively reduces the system to the ideal MHD limit. In the exterior of the star (i.e., $H \approx 1$ ) the anisotropic terms dominate and effectively enforce the force-free condition.

For the initial magnetic field, we adopt a magnetic moment $\mu$ that describes a dipolar magnetic field $\mathbf{B}$ in the comoving frame of the star. This magnetic moment is aligned with the orbital angular momentum. The magnitude of the dipole moment is related to the radial magnetic field at the pole of the star $B_{*}$, by the relation $\mu=B_{*} R_{\mathrm{NS}}^{3}$. The electric field is obtained from the ideal MHD condition $\mathbf{E}=-\mathbf{v} \times \mathbf{B}$, where the velocity in the star is given by the orbital motion, and we assume that the magnetosphere is initially at rest. In our simulations, we take $B_{*}=3 \times 10^{9}$ $\mathrm{G}$ though, as mentioned above, our results can be approximately scaled to arbitrary NS magnetic field values.

The gravitational equations are discretized with fourth-order accurate finite difference operators, while highresolution shock capturing methods based on the HLL flux formula with PPM reconstruction are used to discretize the resistive MHD equations (Palenzuela 2013). The time evolution is performed through the method of lines using a third-order accurate Implicit-Explicit Runge-Kutta integration scheme (Pareschi \& Russo 2005) in order to deal with the stiffness of the resistive equations (Palenzuela et al. 2009). In our production run, the adaptive mesh refinement criteria tolerance is chosen to guarantee that the star is covered by 84 points in each direction. The computational domain $[-1200,1200]^{3} \mathrm{~km}^{3}$ is discretized with seven refinement levels (in addition to the coarsest grid, which covers the whole domain) with a $2: 1$ refinement ratio. The coarsest grid has a grid spacing of $\Delta x=30 \mathrm{~km}$ and the finest grid (which covers the compact objects) has a grid spacing of $\Delta x \approx 0.23 \mathrm{~km}$. We adopt a Courant parameter $\Delta t / \Delta x=0.25$ in each refinement level. To check the consistency of our results, we also evolve (i) the same computational set-up with one less refinement level for the full inspiral and merger; and (ii) another with seven refinement levels, but in which the computational domain extends only to $\pm 600 \mathrm{~km}$ and the coarsest resolution is $\Delta x=20 \mathrm{~km}$, for roughly the first orbit. The results of these additional simulations suggest that our production run is in the convergent regime.

\section{REFERENCES}

Abbott, B. P., et al. 2017a, Phys. Rev. Lett., 119, 161101, doi: 10.1103/PhysRevLett.119.161101

—. 2017b, Astrophys. J. Lett., 848, L12, doi: 10.3847/2041-8213/aa91c9

Abbott, R., et al. 2020a. https://arxiv.org/abs/2010.14533

—. 2020b, Astrophys. J., 896, L44, doi: $10.3847 / 2041-8213 / a b 960 f$

Alic, D., Bona-Casas, C., Bona, C., Rezzolla, L., \& Palenzuela, C. 2012, PhRvD, 85, 064040, doi: 10.1103/PhysRevD.85.064040

Barthelmy, S. D., et al. 2005, Space Sci. Rev., 120, 143, doi: 10.1007/s11214-005-5096-3

Baumgarte, T. W., \& Shapiro, S. L. 2003, Astrophys. J., 585, 930, doi: 10.1086/346104

Bezares, M., Palenzuela, C., \& Bona, C. 2017, Phys. Rev. D, 95, 124005, doi: 10.1103/PhysRevD.95.124005

Blandford, R. D., \& Znajek, R. L. 1977, Monthly Not. Royal Ast. Soc, 179, 433

Buonanno, A., Kidder, L. E., \& Lehner, L. 2008, Phys. Rev. D, 77, 026004, doi: 10.1103/PhysRevD.77.026004

Carrasco, F., \& Shibata, M. 2020, Phys. Rev. D, 101, 063017, doi: 10.1103/PhysRevD.101.063017

Carrasco, F., Shibata, M., \& Reula, O. 2021
Chawla, S., Anderson, M., Besselman, M., et al. 2010, Phys. Rev. Lett., 105, 111101, doi: 10.1103/PhysRevLett.105.111101

Christie, I., Lalakos, A., Tchekhovskoy, A., et al. 2019a, Mon. Not. Roy. Astron. Soc., 490, 4811, doi: 10.1093/mnras/stz2552

Christie, I. M., Lalakos, A., Tchekhovskoy, A., et al. 2019b, Monthly Notices of the Royal Astronomical Society, 490, 4811, doi: 10.1093/mnras/stz2552

Corral-Santana, J. M., Casares, J., Muñoz-Darias, T., et al. 2016, A\&A, 587, A61, doi: 10.1051/0004-6361/201527130

D'Orazio, D. J., Levin, J., Murray, N. W., \& Price, L. 2016, Phys. Rev. D, 94, 023001, doi: 10.1103/PhysRevD.94.023001

Foucart, F. 2012, Phys. Rev. D, 86, 124007, doi: 10.1103/PhysRevD.86.124007

- 2020, Frontiers in Astronomy and Space Sciences, 7, 46, doi: 10.3389/fspas.2020.00046

Gourgoulhon, E., Grandclement, P., \& Novak, J. 2010

HAD home page. 2010

Hansen, B. M., \& Lyutikov, M. 2001, Mon. Not. Roy. Astron. Soc., 322, 695, doi: 10.1046/j.1365-8711.2001.04103.x 
Hawley, J. F., Fendt, C., Hardcastle, M., Nokhrina, E., \& Tchekhovskoy, A. 2015, Space Sci. Rev., 191, 441, doi: $10.1007 /$ s11214-015-0174-7

Ioka, K., \& Taniguchi, K. 2000, Astrophys. J., 537, 327, doi: 10.1086/309004

Lai, D. 2012, Astrophys. J. Lett., 757, L3, doi: 10.1088/2041-8205/757/1/L3

Lee, W. H., \& Ramirez-Ruiz, E. 2007, New J. Phys., 9, 17, doi: 10.1088/1367-2630/9/1/017

Lehner, L., Palenzuela, C., Liebling, S. L., Thompson, C., \& Hanna, C. 2012, Phys. Rev. D, 86, 104035, doi: 10.1103/PhysRevD.86.104035

Lyutikov, M., \& McKinney, J. C. 2011, PhRvD, 84, 084019, doi: 10.1103/PhysRevD.84.084019

McWilliams, S. T., \& Levin, J. 2011, Astrophys. J., 742, 90, doi: 10.1088/0004-637X/742/2/90

Most, E. R., \& Philippov, A. A. 2020, Astrophys. J. Lett., 893, L6, doi: 10.3847/2041-8213/ab8196

Narayan, R., Igumenshchev, I. V., \& Abramowicz, M. A. 2003, Publications of the Astronomical Society of Japan, 55, L69, doi: 10.1093/pasj/55.6.L69

Neilsen, D., Lehner, L., Palenzuela, C., et al. 2011, Proc. Nat. Acad. Sci., 108, 12641, doi: 10.1073/pnas.1019618108

Ortiz, N., \& et al. 2021

Palenzuela, C. 2013, Mon. Not. Roy. Astron. Soc., 431, 1853, doi: 10.1093/mnras/stt311

Palenzuela, C., Lehner, L., \& Liebling, S. L. 2010, Science, 329, 927, doi: 10.1126/science.1191766
Palenzuela, C., Lehner, L., Liebling, S. L., et al. 2013a, Phys. Rev. D, 88, 043011, doi: 10.1103/PhysRevD.88.043011

Palenzuela, C., Lehner, L., Ponce, M., et al. 2013b, Phys. Rev. Lett., 111, 061105, doi: 10.1103/PhysRevLett.111.061105

Palenzuela, C., Lehner, L., Reula, O., \& Rezzolla, L. 2009, Mon. Not. Roy. Astron. Soc., 394, 1727, doi: 10.1111/j.1365-2966.2009.14454.x

Palmer, D. M., Barthelmy, S. D., Lien, A. Y., et al. 2019, GRB Coordinates Network, 25341, 1

Pan, Z., \& Yang, H. 2019, Phys. Rev. D, 100, 043025, doi: 10.1103/PhysRevD.100.043025

Pareschi, L., \& Russo, G. 2005, J. Sci. Comput., 25, 112

Parfrey, K., Beloborodov, A. M., \& Hui, L. 2013, The Astrophysical Journal, 774, 92, doi: 10.1088/0004-637x/774/2/92

Paschalidis, V., Etienne, Z. B., \& Shapiro, S. L. 2013, Phys. Rev. D, 88, 021504, doi: 10.1103/PhysRevD.88.021504

Paschalidis, V., Ruiz, M., \& Shapiro, S. L. 2015, Astrophys. J. Lett., 806, L14, doi: 10.1088/2041-8205/806/1/L14

Piro, A. L. 2012, Astrophys. J., 755, 80, doi: 10.1088/0004-637X/755/1/80

Ponce, M., Palenzuela, C., Lehner, L., \& Liebling, S. L. 2014, Phys. Rev. D, 90, 044007, doi: 10.1103/PhysRevD.90.044007

Ruiz, M., Shapiro, S. L., \& Tsokaros, A. 2018, Phys. Rev. D, 98, 123017, doi: 10.1103/PhysRevD.98.123017

Sathyaprakash, B. S., et al. 2019. https://arxiv.org/abs/1903.09221

Spruit, H. 2008, AIP Conf. Proc., 983, 391, doi: 10.1063/1.2900262

Sridhar, N., Zrake, J., Metzger, B. D., Sironi, L., \& Giannios, D. 2020, doi: 10.1093/mnras/staa3794 